\title{
New Age Detection of Viruses: The Nano-Biosensors
}

\section{OPEN ACCESS}

Edited by:

Hanen Sellami,

Water Research and Technologies Centre (CERTE) of Borj Cedria, Tunisia

Reviewed by:

Sevde Altuntas,

University of Health Sciences, Turkey

Mehrdad Khatami,

Tarbiat Modares University, Iran

*Correspondence:

Suvankar Ghorai suvankarbt@gmail.com

Ramya Devi KT

ramyakanth3@gmail.com

${ }^{\dagger}$ Suvankar Ghorai, 0000-0003-0800-3970;

Ramya Devi KT,

0000-0002-5297-9930

${ }^{\ddagger}$ These authors have contributed equally to this work and share first authorship

Specialty section:

This article was submitted to Biomedical Nanotechnology, a section of the journal

Frontiers in Nanotechnology

Received: 13 November 2021 Accepted: 22 December 2021 Published: 19 January 2022

Citation:

Shand H, Dutta S, Rajakumar S, James Paulraj S, Mandal AK, KT RD

and Ghorai S (2022) New Age

Detection of Viruses: The Nano-

Biosensors.

Front. Nanotechnol. 3:814550. doi: 10.3389/fnano.2021.814550
Harshita Shand ${ }^{1 \neq}$, Swarnab Dutta ${ }^{2 \neq}$, Santhosh Rajakumar ${ }^{3}$, Shalini James Paulraj ${ }^{3}$, Amit Kumar Mandal ${ }^{4}$, Ramya Devi $K T^{3 * t}$ and Suvankar Ghorai ${ }^{1 * t}$

\footnotetext{
${ }^{1}$ Department of Microbiology, Raiganj University, Raiganj, India, ${ }^{2}$ Department of Chemistry, Raiganj University, Raiganj, India, ${ }^{3}$ Department of Biotechnology, School of Bioengineering, Faculty of Engineering and Technology, SRM Institute of Science and Technology, Chennai, India, ${ }^{4}$ Department of Sericulture, Raiganj University, Raiganj, India
}

Viruses and their related diseases have always posed a significant hazard to humans. The current pandemic caused by the Covid-19 (SARS-CoV-2) virus is the latest illustration of what this tiny organism can do to humanity at large, putting everything on the brink of collapse. So it is reasonable that early diagnosis of infection from viruses remains a crucial step to prevent such human suffering. Many traditional methods are already in use for detecting viruses, including molecular approaches, serological methods, direct virus culture methods, and so on. Such traditional methods though are brilliant at some stages but are not devoid of drawbacks. To overcome the limits of conventional procedures, new techniques have been developed which tried to eradicate the demerits of the former procedures. Biosensors have come up with a lot of promises in terms of detecting viruses and diseases connected with them. The development of various types of such biosensors such as Affinity-based nano-biosensors, Nanoisland affinitybased biosensors, Graphene affinity-based biosensors, Nanowires based biosensors, Optical nano biosensors, Fiber optic nano-biosensors, Surface Plasmon Resonance (SPR) based optical nano-biosensors, Total internal reflection fluorescence, Surface-Enhanced Raman Scattering (SERS), Electrochemical nano-biosensors had helped us in the rapid and sensitive detection of viruses. Aid to these nanosensors, viral detection now becomes very sensitive, rapid and cost has come down to a significant low. In this review, an attempt has been made to compile all of the different nano-biosensors and their applications. Due attention is given to the fact that the reader gets the grasp of the concept with much ease.

Keywords: virus, diagnosis, nano, biosensors, SARS-CoV-2

\footnotetext{
Abbreviations: CNT, Carbon Nanotube. EIS, Electrochemical Impedance Spectroscopy; ELISA, Enzyme-Linked Immunosorbent Assay; FRET, Fluorescence Resonance Energy Transfer; HIV, Human Immunodeficiency Virus; HPV, Human Papilloma Virus; HRP, Horseradish Peroxidase; LOD, Limit of Detection; LSPR, Localised Surface Plasmon Resonance; NGS, Next Generation Sequencing; PC: Photonic Crystal; PCR, Polymerase Chain Reaction; QDs, Quantum Dots; RT-qPCR, Quantitative Reverse Transcriptase Polymerase Chain Reaction; SARS-CoV-2, Severe Acute Respiratory Syndrome Corona Virus 2; SAW, Surface Acoustic Wave; SERS, Surface-Enhanced Raman Spectroscopy; SPR, Surface Plasmon Resonance; SWLNTs, Single Walled Carbon Nanotubes; TIRF, Total Internal Reflection Fluorescence.
} 


\section{INTRODUCTION}

Viruses can infect a wide range of hosts, including humans, poultry animals, plants, insects, and even microbes. If the viral infection is not recognized early, it can cause serious diseases in the host, most of which are fatal. Molecular approaches, serological methods, direct virus culture methods, and other traditional methods have been used to detect viruses since the beginning. Culture technique is one of the most significant virology methods for determining the viral titer or the amount of virus in a sample. This is mostly a direct approach for detecting viruses. Plaque assays focus on forming assays, and transformation assays are used to determine these characteristics features. In the Plaque assay, the monolayers of cultured cells are incubated with the preparation of the virus to allow adsorption to the cell. When the originally infected cells release progeny particles, the gel restricts the spread of viruses to neighboring uninfected cells. As a result, each infectious particle produces a clear zone of infected cells called plaque. Focus forming assay can easily detect the advancement of various materials, particularly nanomaterials such as carbon nanotubes, graphene, carbon nanoparticles, magnetic and metal nanoparticles, nanosized clays, and different polymers such as nanosized and nanostructured polymers, molecularly imprinted polymers, and hybrids. Transformation assay is also known as the fluorescent focus test, which is a variation of the plaque assay. The transformed cells from tiny stacks or foci after infection can be easily distinguished from the rest of the uninfected cells in a mono-layered culture plate (Cooper, 1955). In Serological diagnosis, we can assess the concentration of antigen or antibodies by performing these procedures. In most serological tests, we utilize fast antigen assays, which are tiny kit that allows us to detect whether a certain antigen is present or not in a patient's serum. ELISA is used to identify any antigens or antibodies in a patient's serum sample. ELISA has several drawbacks, including the fact that it can be more difficult to quantify enzyme activity, that which is influenced by plasma components, so it can provide false positive and negative results, and also it is expensive (Clark et al., 1986). RIA is utilized to examine a chemical or antigen that's only present in trace amounts. Here, radio-labelled and unlabelled antigens compete for binding to a high-affinity antibody (Bauxbam, 2011). Rapid Test Kit is also based on antigenantibody reaction. Nowadays, this has wide use in the detection of Coronavirus. But these give false positive or negative results. The molecular diagnostic can easily detect with the advancement of various materials, such as carbon nanotubes, graphene, carbon nanoparticles, magnetic and metal nanoparticles, nanosized clays, and different polymers such as nanosized and nanostructured polymers, molecularly imprinted polymers, and hybrids. Rapid Test Kit with the progress of numerous materials, such as carbon nanotubes, graphene, carbon nanoparticles, magnetic and metal nanoparticles, nanosized clays, and diverse polymers such as nanosized and nanostructured polymers, molecularly imprinted polymers, and hybrids, we can readily detect for future use (Gu and Zhang, 2018).

\section{NANO-BIOSENSORS}

A nano-biosensor consists of a receptor transducer and detector that generate a digital output. The transducer then converts the signal analysed by the detector. The efficiency of nanosensors can be improved by employing nano-films, gold nano-particles (La Spada and Vegni, 2018) quantum dots for the amplification of signals. Nanomaterials are known to have excellent optical, electrical, magnetic, mechanical properties and a uniquely high surface area over volume ratio that can improve patient care as it provides technique with minimal invasion and can provide rapid, sensitive and specific detection of viruses at even low level.

Nanoparticles can be classified into different classes based on their size, shape and structure. NPs possess distinctive physical and chemical properties because of their high surface area and nanoscale size. The sensitivity of a sensor is enhanced by employing nano-particles. For example, the lowest LOD of H7 subtype Influenza A virus by ELISA is $10 \mathrm{ng} / \mathrm{ml}$ and the LOD of LSPR- induced immunofluorescence nanobiosensor for influenza virus is $0.4 \mathrm{pg} / \mathrm{ml}$. Their reactivity and toughness are also a result of their size, shape and structure. There are different types of nanosensors. Affinity-based nanobiosensors are a type of biosensors that interact with receptors such as ssDNA, antibodies, and aptamers interact with nanoparticles in affinity-based nano biosensors to offer high sensitivity (Bishop et al., 2009).

The increased surface/volume ratio enables efficient selective surface contact of nano-biosensor with biotinylated, resulting in bio-receptor immobilization. Affinity-based biosensors have been coupled with different nanoparticles such as gold nanoparticles, graphene, and nanowires to detect SARSCoV2 in recent times due to the global coronavirus outbreaks (Drobysh et al., 2021). Optical nanosensors employ gold nanoparticles because they improve the refractive index change. Gold nanoparticles' binding ability has been improved by conjugating them with carbon nanotubes (CNTs), which offer better and more effective immobilization. Gold nano-islands are gold aggregates ranging in size from 20 to $80 \mathrm{~nm}$ and are also utilized in the development of biosensors.

Graphene possesses excellent electrical, thermal, and mechanical properties and it is integrated with an electrochemical biosensor for improved detection of bioanalysts (Figure 1A). It is also cost-effective, chemically stable, electrochemically inert, and shows excellent conductivity. It shows efficient immobilization to ligands like ssDNA and antibodies, and this can be achieved by employing techniques like covalent-binding, electro-polymerization, and adsorption techniques. Graphene is also used in optical and field-effect transistors (FET) (Wu et al., 2018).

Nanowires have a diameter comparable to that of the biotinylated that are to be analyzed. NWS possesses highly reproducible electrical as well as optical properties (Duan et al., 2001; Bishop et al., 2009). Silica Nanowires are widely used in biosensing as it shows great photonic properties; it also has high biocompatibility for sensing purposes (Ambhorkar et al., 2018). In an aqueous solution, biological molecules are charged and can be easily detected if NWs are tagged with suitable 

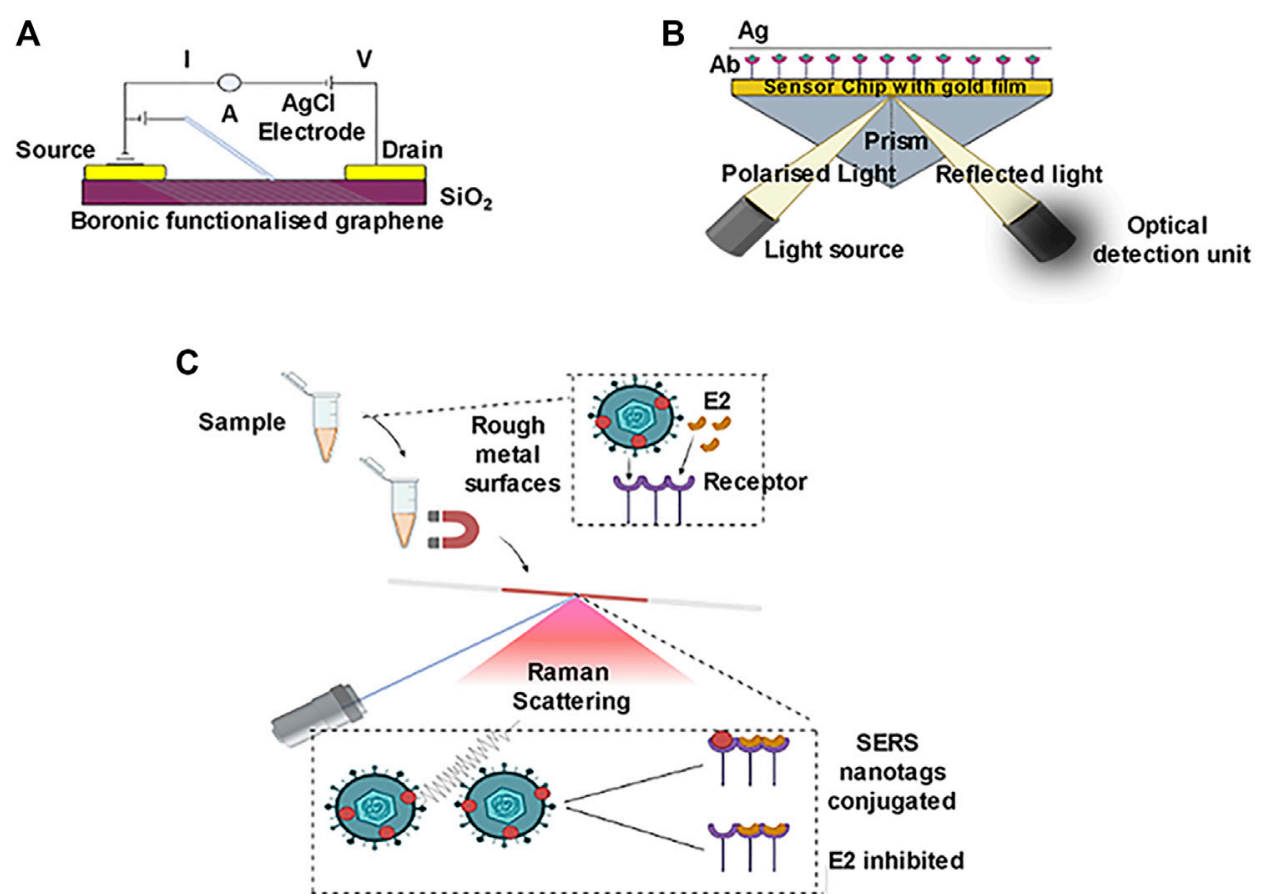

FIGURE 1 | Graphene affinity based biosensor. Surface plasmon resonance (SPR) based biosensor. Surface enhanced raman scattering.

receptors. SiNWs can be used to detect viral particles with high sensitivity (Kaushik et al., 2014). An optical nano-biosensor is referred to as a sensor that uses light to detect the effect of chemicals on a biological system. The principle for this kind of nano-biosensor mainly relies on optical and luminescent properties of nanoparticles, luminescence resonance energy transfer, and surface plasmon effects. QDs, UCNPs, AuNPs are the nanomaterials used as well as organic dye-based nanoparticles are used in biosensors that have a capacity of absorption of luminescence. This kind of biosensor is well designed to accept the fluorescence signal when targeting the analytes (Campion and Kambhampati, 1998; Gu and Zhang, 2018). Fiber-optic nano-biosensors (also named optrodes) are devices having an immobilized biocatalyst fixed at the distal end of a fiber-optic detection device (Leung et al., 2017). This biocatalyst mediates between a sensor and an analyte, assisting the detection of a desirable compound from a sample of interest. They are also used in agricultural sectors to quantify pesticides using the principle of conical-shaped optical fiber detection (Arjmand et al., 2017). Surface plasmon resonance (SPR) -based optical nano-biosensor follows an optical measurement of refractive index change using monochromatic light that excites the plasmons (Prabowo et al., 2018) (Figure 1B). These types of biosensors can detect large molecules, hence are considered universal detectors. SPR based optical nano biosensors are used in clinical research, mainly in quantifying drugs requiring TDM, such as anticoagulants, antibiotics, and anticancer drugs. There are two variations in this SPR phenomenon, such as SPR imaging (SPRi) and localized surface plasmon resonance (LSPR) (Ktari et al., 2011). Total Internal
Reflection Fluorescence (TIRF) nano-biosensors are based on the fluorescence activity of nanomaterials. This type of biosensor is used especially for the environmental monitoring of wastewater from industries that could affect human and animal health and also find great use in quantifying immunosuppressant drugs. In Surface-enhanced Raman scattering (SERS), amplifying the intensity of the Raman scattering phenomenon by using NPS or metallic structures is the main principle of the SERS technique (Garzón et al., 2019) (Figure 1C). This type of biosensor is used to quantify drugs as well as to detect antibiotics. Electrochemical biosensors are biosensors where direct conversion of a biological constituent to an electrical signal is performed (Figure 2). The advantages of this method include enhanced detection levels, ability to employ turbid bio-fluids, and rapid measurement. The approach for immobilization of the particular recognition elements demands their interactions with transition metal complexes on the electrode surface, which was primarily based on the redox-active monolayer. Electrochemical nano biosensors are the most attended form of biosensors for their low-cost and less time-effective procedure as well as higher sensitivity and lower sample quantity needed (Shoaie et al., 2019). Electrochemical detection techniques include voltammetric, amperometric, impedimetric, and potentiometric strategies.

The voltammetric method is a type of electrochemical nanobiosensors; the current is measured as a function of electrode potential that results in the quantitative determination of ions and molecules. This technique has fewer limitations than other techniques (Schloz, 2015). 


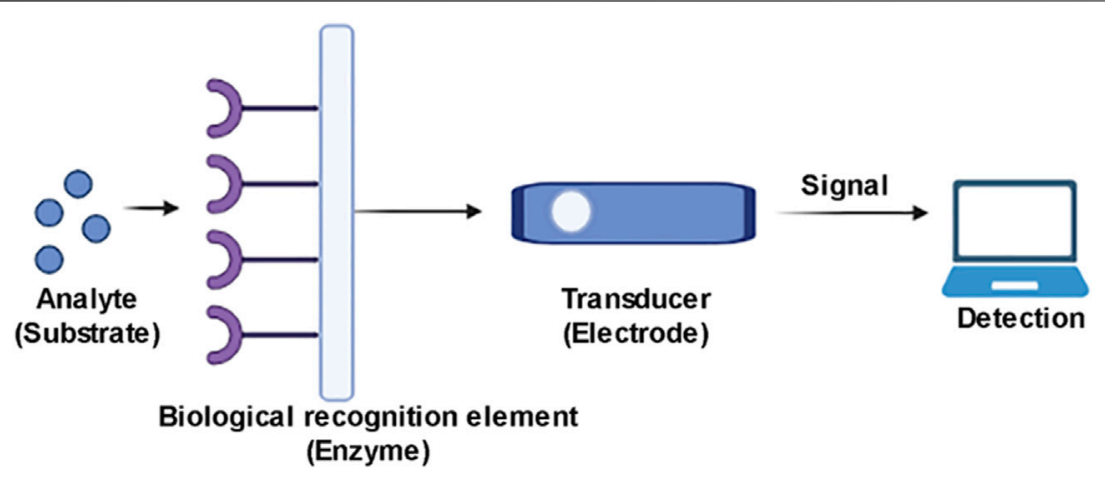

FIGURE 2 | Electrochemical nanobiosensor for viral detection.

In Amperometric, current in electrodes is measured based on the redox activity of an enzyme (mainly horseradish peroxidase/ HRP) (Davis and Higson 2012; Bollela and Gorton, 2018; Monteiro and Almeida, 2019). Enzymes can accelerate chemical reactions due to their catalytic activity and specific binding capacity. Although enzymes have drawbacks, including limited stability, tendency to lose activity after a relatively short time, functioning capacity, and expensive source and extracting, isolating, purifying process (Shoaie, et al., 2019). In the impedimetric method type of electrochemical nano-biosensors, the impedance of a system is measured over a range of frequencies. Electrochemical impedance spectroscopy (EIS), the most important technique over other methods, can be performed as a label-free detection method (Bahadır and Sezgintürk, 2016; Xu et al., 2016). Potentiometric method electrochemical nano-biosensor gauges the charge accretion (potential) between an ion-selective electrode and a reference electrode. Magnetic biosensors have gained great attention in the past 2 decades. Surface and volume-based magnetic biosensors have been made to detect viruses, pathogens, cancerous biomarkers (Aytur et al., 2006; Rettcher et al., 2015). The benefit of a magnetic nano-biosensor over an optical or electrochemical biosensor is that it creates less background noise because the biological environment is mainly non-magnetic.

\section{Detection of Human Immuno Deficiency Virus}

There are mainly two serotypes of HIV, HIV-1 (Global prevalence) and HIV-2 (West Africa prevalence). AIDS is mainly a sexually transmitted disease but non-sexual transmission can occur from mother to her infant during pregnancy and also during labour by exposure to her blood or vaginal fluid and even through her breast milk (Singh et al., 2016). $\mathrm{HIV}$ is a positive-sense single-stranded RNA virus with a genome size of around $9 \mathrm{~kb}$. Generally, the incubation period of this virus ranges from several weeks to several months, and the viral load is undetectable during this phase. There are several standard diagnostics available for HIV like RT-PCR, ELISA and rapid test. The nucleic acid hybridization strategies for rapid test have some disadvantages such as failure to produce precise results in acute or severe HIV diagnosis as there is a need for quantification of RNA from the HIV (La Spada and Vegni, 2018), DNA electrochemical based test measures used for HIV detection, which involves the hybridization approach of HIVcomplementary DNA fragments present in the sample is still in the research phase and needs to be modified to make more accurate, precise, and rapid for larger-scale use in the diagnostic laboratories (Wang et al., 1996; Bishop et al., 2009). Surface Plasmon Resonance (SPR) which is based on the variation of the solvent's refractive index during complex formation and dissociation, may detect the presence of HIV viruses and show a lot of promise for future diagnostics. This sort of biosensor can identify a variety of bio-molecules, including viral particles, proteins, and RNA. Improved piezoelectric biosensors are more appealing HIV detection methods, the screening test is done by BIOTEST AG, having a 100\% specificity. Photonic crystal (PC) nanostructured optical biosensors are also used to detect HIV. 384 well microplates with an elastic polyester substrate are utilized for PC biosensors. For HIV diagnosis, this method is quite effective. Sensitive impedimetric biosensors are based on impedimetric analysis that consists of super magnetic particles or magnetic beads which are used as a label for HIV diagnosis. Polyethyleneimine magnetic beads can also be used for this technique (Guily et al., 2019).

\section{Detection of Influenza Virus}

Influenza, sometimes known as the flu, is caused by the influenza virus, which belongs to the Orthomyxoviridae family. This virus affects around two million individuals each year. Fever, running nose, sore throat, muscular discomfort, headache, coughing, and tiredness are some of the symptoms. If not diagnosed early, this might result in a serious condition. The most often used techniques for viral detection include real-time polymerase chain reaction, enzyme-linked immunosorbent assay, and reverse transcription-polymerase chain reaction (Cooper, 1961). But all of them have their own limitations. A new-age method to detect the Influenza virus has been extensively exploited. Antibody-modified boron-doped diamond biosensors are one such example. Boron-doped diamond electrode biosensor is a technique that uses a sialic acid-mimic 


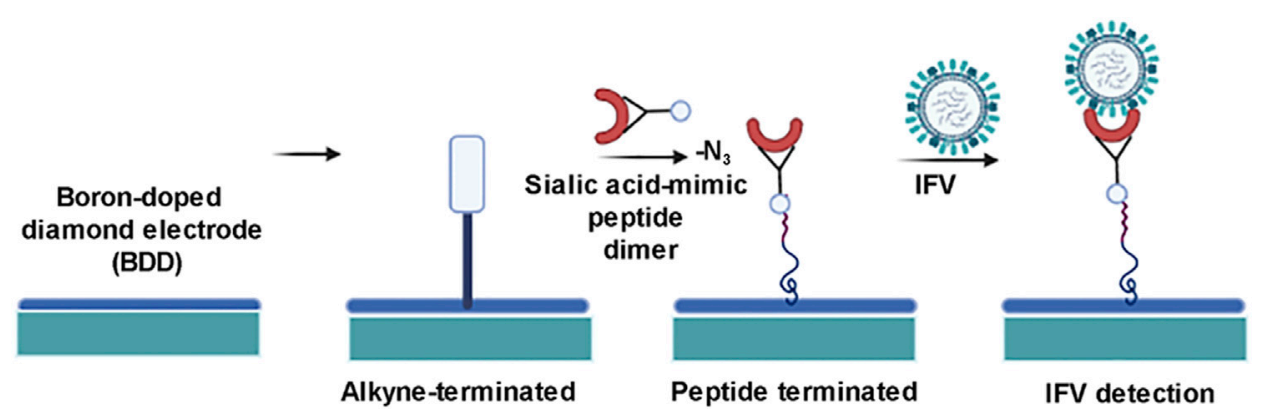

FIGURE 3 | Schematic diagram for detection of influenza virus by boron doped diamond (BDD) nanobiosensor.

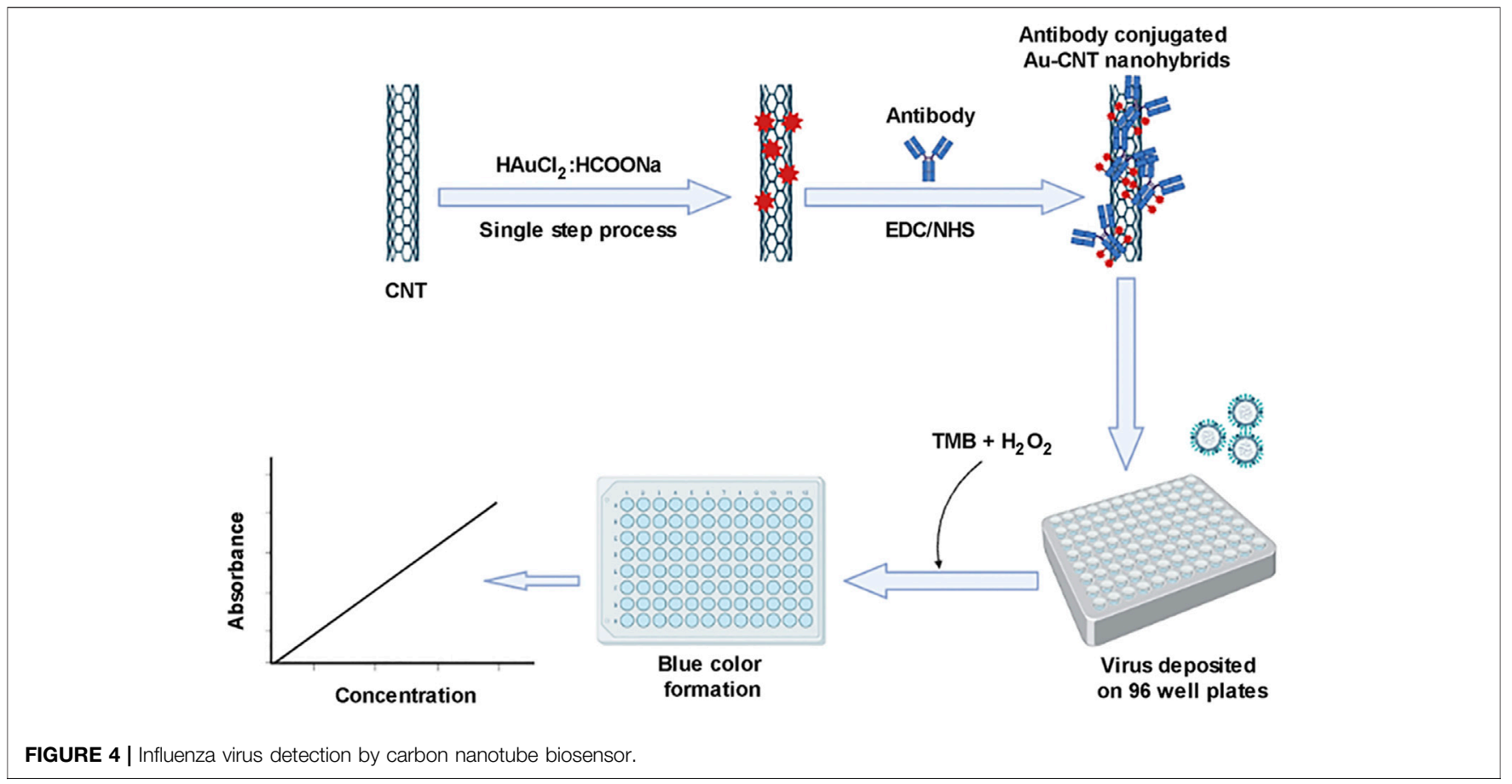

pentapeptide using click-chemistry reaction for the detection of influenza virus (Figure 3). The peptide is used instead of the more common silyl oligosaccharide receptor 8,9 , which is the common receptor for influenza $\mathrm{A}$ and $\mathrm{B}$. $\mathrm{H} 1 \mathrm{~N} 1$ and $\mathrm{H} 3 \mathrm{~N} 2$ IFVs were detectable using impedance spectroscopy, in the range of 20-400 plaque-forming units (pfu) after $45 \mathrm{~min}$ of incubation. This sensor is a form of nanoscale surface biosensor that detects anti-M antibodies and allows for an order of magnitude improvement in detection speed and resolution for early detection of influenza virus. A sample of the throat or nasal wash is collected for early viral identification. The detection of influenza $\mathrm{A}$ and $\mathrm{B}$ viruses is also done by using a quartz crystal microbalance biosensor. The mass sensitivity of the immunosensor was enhanced by combining $13 \mathrm{~nm}$ gold nanoparticles with the detecting antibody, resulting in a 10 fold improvement in sensitivity and a detection limit of $1 \times$ $10^{3} \mathrm{pfu} / \mathrm{ml}$. This method is less expensive than PCR and ELISA.
The nasal wash is used to collect the sample (PeduruHewa et al., 2009).

Carbon nanotube electric immunoassay is used for the detection of swine influenza virus $\mathrm{H} 1 \mathrm{~N} 1$, this biosensor is based on the electrical properties of single-walled carbon nanotubes (SWCNTs). Here, the virus and the antibody complexes influence the conductance of SWCNT thin film. This thin film has been constructed by facile layer by layer self-assembly. The final step of this essay is done by using the electric characterization of immunochips. The resistance of immunochips tends to increase upon surface adsorption of macromolecules such as poly-L-lysine, anti-SIV antibodies, and SIVs tend to improve immunochip resistance. This resistance develops when SIV binds to anti-SIV. Non-SIVs are used in the sensor selectivity experiments, which exhibit a normalized resistance shift of $12 \%$ as a background (Lee et al., 2011) (Figure 4). 


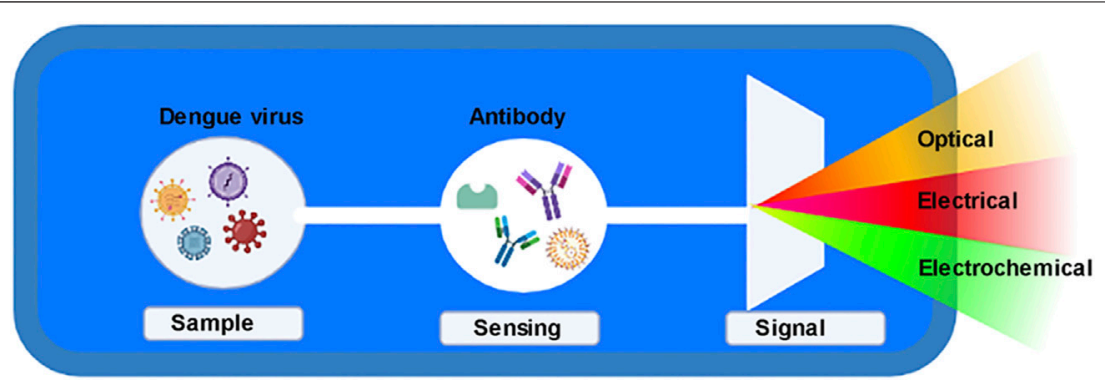

FIGURE 5 | Dengue virus detection.

Optical biosensors are used for sensitive detection of influenza virus with genetically engineered fusion protein by combining microarray and manufacturing nanoparticles onto a single glass chip; a full optical analytical system was established, enabling specific and sensitive diagnosis with subsequent binding. SPR analysis was used to optimize reaction conditions for maximal reactivity, while SPRi analysis was used to perform more sensitive interactions. By switching manufacturing procedures created on the gold surface utilizing nanoparticles, the advanced phase-in of the enhanced plasmonic sensing system enables more efficient and sensitive detection. Another novel approach for detecting the influenza virus in swabs from the human throat is electrochemical spectroscopy. This technique detects all kinds of influenza A virus quickly and with a sensitivity equivalent to molecular approaches. Detection of avian influenza virus subtype $\mathrm{H} 5$ using a biosensor based on high spatial resolution image ellipsometry has been described as a new technique. The resulting alterations on the wafers' surface were immediately observed in gray-scale on an imaging ellipsometry picture. This biosensor not only has higher sensitivity than lateral-flow immunoassays, but it can also perform multiplexed tests at the same time and have opened up a new dimension for the development of different biosensors (Qi et al., 2010).

\section{Detection of Dengue Virus}

Dengue virus, the causative agent of dengue fever is indeed a matter of concern because there are no antivirals and vaccines present to date to control the Dengue virus. Rapid and fast detection is thus the need of the hour.

Optical biosensors are used for dengue virus detection as they generally measure photons rather than electrons. The residue-free nucleic acid immobilization along with microfluidic functionalization enables it to detect the Dengue virus in multiplexed mode (Boonham et al., 2014). Surface Plasmon resonance (SPR) based biosensor is one of the first optical label-free biosensors for detecting the Dengue virus. The SERS (Surface-enhanced Raman scattering) biosensor is primarily based on sensitive optical detection for the early detection of viral illnesses such as Dengue fever, but it can also be used to detect cancer. The Raman bands are too simple here, with a simple fingerprint sensor that simplifies the procedure and can also be studied using SERS spectra. Photobleaching issues aren't a problem in this area. This is useful for detecting the Dengue virus, and it's done on the surface of a SERS active Ag-Au bimetallic device that has been functionalized with DNA probes (Ngo et al., 2014). This is a very effective method of detecting Dengue fever early on. For viral detection, this technique is inexpensive and practical. On the sensor surface, the Dengue Ag-antibody binding interaction was directly proportional to target concentration. Sensor antibodies bind to antigens that have been fixed on the biosensor's surface as probes in this test. Temperature and optical control, as well as the size of these sensors, pose significant challenges (Figure 5).

Microfluidics systems based sensors are also tried for dengue virus detection. Microfluidics is a multipurpose technology that combines several technologies. This also has advantages such as reduced reagent use, little material handling, and a quick test time (Kabir et al., 2021).

A combination of polymethacrylate microspheres and a microfluidic device sensitive to Dengue virus antibodies was used to speed up the experiment. The sensor was able to detect 20 and 25 long capture oligonucleotides with a LOD value of $10 \mathrm{pmol}$ after being enhanced by a liposomal encapsulated fluorescent reagent (Lee et al., 2009).

A novel method for detecting the Dengue virus uses a metal oxide-based electronic biosensor. These devices function by making contact between a biomolecule and a metal oxide layer and then transferring charge from the biomolecule to the underlayer, which generates an electrical signal. The sensitivity of these biosensors is highly dependent on the specific region of the underlayer (Garzón et al., 2019).

Because of their cost efficiency, ease of availability, and quick detection systems for a variety of electroactive analytes, voltammetric techniques might be used in a wide range of biosensors. One of the appealing aspects of DNA biosensors is their ultra-sensitivity, which allows for early detection of the Dengue virus. The capture probe's immobilization method can potentially affect biosensor performance. The measuring of current as a result of the reduction/oxidation of an electroactive label or tag employed in Amperometric biosensors. This method aids in the early identification of the Dengue virus.

\section{Detection of Human Papilloma Virus}

Human Papilloma Virus (HPV) is responsible for causing Gynaecological malignancy, Cervical Cancer- a type of 
TABLE 1 | Different biosensors for the detection of Viruses and their properties.

\begin{tabular}{|c|c|c|c|c|}
\hline Virus name & Methods & Expertise level & Limit of detection & References \\
\hline \multirow[t]{4}{*}{ HIV } & SPR(Surface plasmon resonance) based nano-biosensor & Professionals & $48 \mathrm{fM}$ & Diao et al. (2018) \\
\hline & DNA electrochemical based nano-biosensor & Professionals & $0.3 \mathrm{fM}$ & Babamiri et al. (2018) \\
\hline & Piezoelectric nano-biosensor & Professionals & $2 \mathrm{mg} / \mathrm{ml}$ & Lu et al. (2012) \\
\hline & Sensitive impedimetric nano-biosensor & Professionals & $3.0^{\star} 10^{-13} \mathrm{M}(\mathrm{S} / \mathrm{N}=3)$ & Gong et al. (2015) \\
\hline \multirow[t]{4}{*}{ Influenza } & Antibody modified boron doped diamond nano-biosensor & Professionals & $1 \mathrm{fg} / \mathrm{ml}$ & Nidzworski et al. (2017) \\
\hline & Quartz crystal microbalance nano-biosensor & Professionals & $1103 \mathrm{PFU} / \mathrm{ml}$ & PeduruHewa et al. (2009) \\
\hline & Carbon nanotube electric immunoassay & Professionals & $180 \mathrm{TCID}_{50} / \mathrm{ml}$ OF SIV & Lee et al. (2011) \\
\hline & Optical nano-biosensor & Professionals & $2-100 \mathrm{ng} / \mathrm{ml}$ & Pang et al. (2015) \\
\hline \multirow[t]{3}{*}{ Dengue Virus } & Optical nano-biosensor & Professionals & $0.125 \mathrm{nM}$ & Zaytseva et al. (2005) \\
\hline & Electrochemical nano-biosensor & Professionals & $0.92 \mathrm{nM}$ & Souza et al.,. (2011) \\
\hline & Piezoelectric nano-biosensor & Professionals & 2 PFU.ml $\mathrm{ml}^{-1}$ & Chen et al. (2009) \\
\hline \multirow[t]{3}{*}{ HPV } & Electrochemical nano-biosensor & Professionals & 1 attomole & Wang et al. (2013) \\
\hline & FRET technique based nano-biosensor & Professionals & $0.2 \mathrm{~nm}$ & Shamsipur et al. (2017) \\
\hline & Surface acoustic wave biosensor & Professionals & $1.21 \mathrm{pM}$ & Wang et al. (2009) \\
\hline \multirow[t]{2}{*}{ SARS Cov-2 } & Graphene based FT device & Professionals & 3.99F m & Li et al. (2021) \\
\hline & SERS based aptasensors & Professionals & 10 PFU.ml ${ }^{-1}$ & Chen et al. (2021) \\
\hline
\end{tabular}

fM-femtomolar, mg-Milligram, ml-Milliliter, M-Molar, Fg-Femtogram, PFU-plaque forming assay, TCID 50 -Median Tissue Culture Infectious Dose, SIV-swine influenza virus, ng-Nanogram, nM-Nanomolar, pM-picomolar, S/N-signal by noise ratio.

malignant tumour that occurs in the cervix, the lowermost part of the uterus, which is in turn connected to the vagina. When exposed to HPV, the virus can survive for years, contributing to a process that causes the cervical cells to transform into cancerous cells. HPV Type-16 and Type-18 cause $70 \%$ of cervical cancer and pre-cancerous lesions. The current Diagnostic Approach for HPV includes, Paps-smear Test, which has a false negative rate of $13-70 \%$ and that of false positive is $0-14 \%$. Technique like microarray, quantitative Reverse Transcription Polymerase Chain Reaction (qRT-PCR) and next-generation sequencing (NGS) is highly specific and sensitive but they are extremely expensive and time-consuming.

Electrochemical nano-sensors provide sensitive selective and specific detection technique as an alternative to pre-existing technique. It employs Cyclic Voltammetry, Square wave Voltammetry (SWV), Electrochemical Impedence Spectroscopy (EIS) to measure the output signal generated.

Wang et. Al in 2013, employs the EIS technique and developed the Electrochemical Sensors by fixing gold nanoparticles and immobilising ssDNA probe on SWCNT platforms. This technique provides a highly specific biosensing platform for HPV Type-16 detection with a limit of one attomole (Wang et al., 2013). With the advancement in nanotechnology, giantmagneto resistive biochip provides an alternative way to detect HPV. These biochips are based on magnetic nanoparticle and spin valve sensor array which provides cost effective and sensitive approach for HPV DNA detection. It can detect the viral DNA at a concentration as low as $10 \mathrm{mP}$ (Xu et al., 2008).

Fluorescence based nano-biosensors can serve as an efficient tool in HPV detection. Fluorescence labelled probe allows multiplex detection in a single assay at low cost and rapidly.

Shamsipur et al., in 2017 developed a nano biosensor based on FRET technique for the rapid detection of 22-mer Oligonucleotide of HPV Type-18 gene using water soluble CdTe quantum dots. It has a detection limit of $0.2 \mathrm{~nm}$
(Shamsipur et al., 2017). Zhang et al. used microbeads to detect the target DNA molecule of HPV Type-16 and type-18 (Zhang et al., 2011). Surface acoustic wave (SAW) biosensors detect biomarkers by using acoustic waves. Acoustic waves are confined on microfabricated sensors to detect HPV target DNA molecules hybridised on the surface to detect target DNA molecules. Its LOD is 1.21 pM (Wang et al., 2009).

\section{Detection of SARS Cov-2 Virus}

The novel coronavirus which was detected in Wuhan, PCR in December 2019 has caused the global pandemic outbreak, which we are currently struggling with. To get a hold of this pandemic, rapid and sensitive diagnostic technique is the need of the hour. For the most part, Covid-19 detection is based on quantitative Reverse transcriptase Polymerase Chain Reactions (RT-PCR). But the requirement of sophisticated instruments and trained manpower limits its use especially in a low resource setting area. The other techniques that are being employed for detection are less efficient and show low accuracy (Lisboa Bastos et al., 2020). For rapid diagnosis of viral particles, nano-biosensors are being developed. To determine the severity of the disease graphene based FET devices have been developed which helps to detect the SARS-COV-2 viral load in the nasopharyngeal swabs of patients (Seo et al., 2020). A graphene sheet attached to $\mathrm{SiO}_{2} / \mathrm{Si}$ substrate is present in the screening area of FET- based biosensor which is modified with SARS-COV-2 antibody. This is a highly sensitive technique that helps to determine as low as $1 \mathrm{fg} / \mathrm{ml}$ in phosphate buffer which is much lower than the value provided by ELISA and PCR (Chu et al., 2020). FET biosensor showed discrimination between healthy and diseased samples with lower detection limits as compared to the currently used methods without any sample preparation. It also showed high accuracy and sensitivity as it doesn't respond to MERS- CoV spike proteins.

Qiu and coworkers have recently developed a DNAnanosensor (Qiu et al., 2020). It consisted of a DNA-sensor 
with a single chip compiled with gold nano-island (Au NIs) plasmonic photothermal (PPT) effect and localised surface plasmon resonance (LSPR) was combined by the chip for sensing transduction.

Optic-based nanobiosensors employ light energy for the detection of changes in any biological system and have recently proved as a boon in Covid-19 pandemic times. SERS is an example of optic based nanobiosensor which amplifies the intensity of Raman scattering phenomenon by using nanoparticles or metallic structures. SERS-based aptasensors has been recently developed for detection and quantification of SARS-CoV2 in biological sample with high sensitivity and specificity. Its limit of detection is less than $10 \mathrm{PFU} / \mathrm{ml}$ and time of detection is $15 \mathrm{~min}$.

SERS based aptasensors detect the SARS-CoV2 spike proteins by employing DNA-aptamers supported on AU-nanopopcorn surfaces. As the spike protein binds the aptamers, it moves away from $\mathrm{Au}$ nanopopcorn surface which leads to a decrease in Raman peak intensity of Cy3 reporters (Chen et al., 2021). Nano-Biosensors corresponding to 5 relevant viruses are summarised in a tabular form (Table 1).

\section{CONCLUSION}

Virus-related diseases constitute a public health concern and the recent pandemic caused by SARS-CoV-2 has proved again that viral infections are a threat to the very existence of life on Earth. As the eclipse period of the virus is normally asymptomatic, healthcare seeking tendency is poor in infected individuals and by the time the symptom appears and the virus has been detected, the virus has already taken control over the host immune system. Viruses, especially RNA viruses, can develop very quickly over time, rapid detection is very much essential to keep them under control. The conventional molecular methods for the detection of viruses are always been the gold standard but it is sophisticated machine-dependent and trained personnel are required. Sometimes it takes quite a significant amount of time and hence alternative methods especially in low resource area are always a need.

Nanotechnology has come up with a great promise in developing biosensors. Biosensors have been used to create a variety of detection techniques, including optical biosensors, photonic crystal biosensors, colorimetric biosensors, SPR biosensors, fluorometric biosensors, and electrochemical biosensors. Because of various benefits such as high sensitivity, selectivity, repeatability, cheap cost, minimal sample need, and a

\section{REFERENCES}

Ambhorkar, P., Wang, Z., Ko, H., Lee, S., Koo, K.-i., Kim, K., et al. (2018). Nanowire-Based Biosensors: From Growth to Applications. Micromachines 9 (12), 679. doi:10.3390/mi9120679

Arjmand, M., Saghafifar, H., Alijanianzadeh, M., and Soltanolkotabi, M. (2017). A Sensitive Tapered-Fiber Optic Biosensor for the Label-free Detection of tiny device that can be utilized with simple handling and operation, the notion of biosensors has been gaining traction among the general public. This review takes advantage of newly discovered biosensors for managing viral illnesses. Nanobiosensors have a bright future in biomedical research since the detection is more accurate, less time-consuming, and costeffective.

\section{FUTURE ASPECT OF NANO-BIOSENSOR}

Virus is a threat to mankind as they have a wide range of host and can cause both acute and chronic illness. AIDS, Dengue, Influenza, Hepatitis and presently the Covid-19 are some of the examples of catastrophic viral diseases that can severely impact every aspect of human life. Therefore, it is of utmost importance and indeed the need of the hour that an early, rapid and sensitive technique for recognition and quantification is develops in order to curb this menace of viral infection. Over the past decades, research has been conducted to come up with such a technique. Nanotechnology has the potential to rapidly identify viruses. Nanoparticles used in the nano-biosensors have unique optical, magnetic, electronic, mechanical properties that can lead to accurate detection of viruses with increased precision and selectivity. In this review, we have described various nanobiosensors for the detection of pathogenic viruses like HIV, Influenza, Dengue, HPV and SARS-CoV-2. The use of graphene oxide, quantum dots, silica, gold, magnetic nanoparticles gives a suitable and accurate platform to detect the viral components effectively. This review focuses on the advantages of nano-biosensor over the existing conventional techniques to detect pathogenic viruses. Researchers from all over the world are giving great efforts to make the portable and reusable devices for sensitive detection of viral components. Nano-biosensor in the near future will be a major breakthrough in diagnosis as they will provide early detection of diseases with unprecedented precision that will improve the health care system and provide patients with the best care possible.

\section{AUTHOR CONTRIBUTIONS}

HS: Manuscript writing, formatting, submitting SD: Manuscript writing, formatting, submitting SG: Overall supervision RD: Overall supervision SP: Image construction and editing SR: Image construction and editing AM: Overall supervision.

Organophosphate Pesticides. Sensors Actuators B: Chem. 249, 523-532. doi:10.1016/J.SNB.2017.04.121

Aytur, T., Foley, J., Anwar, M., Boser, B., Harris, E., and Beatty, P. R. (2006). A Novel Magnetic Bead Bioassay Platform Using a Microchip-Based Sensor for Infectious Disease Diagnosis. J. immunological Methods 314 (1-2), 21-29. doi:10.1016/j.jim.2006.05.006

Babamiri, B., Salimi, A., and Hallaj, R. (2018). A Molecularly Imprinted Electrochemiluminescence Sensor for Ultrasensitive HIV-1 Gene Detection 
Using EuS Nanocrystals as Luminophore. Biosens. Bioelectron. 117, 332-339. doi:10.1016/j.bios.2018.06.003

Bahadır, E. B., and Sezgintürk, M. K. (2016). A Review on Impedimetric Biosensors. Artif. Cell nanomedicine, Biotechnol. 44 (1), 248-262. doi:10.3109/21691401.2014.942456

Bauxbam, E. (2011). "Immunological Methods," in Biophysical Chemistry of Proteins (Springer), 97-122. doi:10.1007/978-1-4419-7251-49

Bishop, K. J. M., Wilmer, C. E., Soh, S., and Grzybowski, B. A. (2009). Nanoscale Forces and Their Uses in Self-Assembly. Small 5 (14), 1600-1630. doi:10.1002/ smll.200900358

Bollella, P., and Gorton, L. (2018). Enzyme Based Amperometric Biosensors. Curr. Opin. Electrochemistry 10, 157-173. doi:10.1016/j.coelec.2018.06.003

Boonham, N., Kreuze, J., Winter, S., van der Vlugt, R., Bergervoet, J., Tomlinson, J., et al. (2014). Methods in Virus Diagnostics: from ELISA to Next Generation Sequencing. Virus. Res. 186, 20-31. doi:10.1016/j.virusres.2013.12.007

Campion, A., and Kambhampati, P. (1998). Surface-enhanced Raman Scattering. Chem. Soc. Rev. 27 (4), 241-250. doi:10.1039/A827241Z

Chen, H., Park, S.-G., Choi, N., Kwon, H.-J., Kang, T., Lee, M.-K., et al. (2021). Sensitive Detection of SARS-CoV-2 Using a SERS-Based Aptasensor. ACS Sens. 6 (6), 2378-2385. doi:10.1021/acssensors.1c00596

Chen, S.-H., Chuang, Y.-C., Lu, Y.-C., Lin, H.-C., Yang, Y.-L., and Lin, C.-S. (2009). A Method of Layer-By-Layer Gold Nanoparticle Hybridization in a Quartz crystal Microbalance DNA Sensing System Used to Detect Dengue Virus. Nanotechnology 20 (21), 215501. doi:10.1088/0957-4484/20/21/215501

Chu, D. K. W., Pan, Y., Cheng, S. M. S., Hui, K. P. Y., Krishnan, P., Liu, Y., et al. (2020). Molecular Diagnosis of a Novel Coronavirus (2019-nCoV) Causing an Outbreak of Pneumonia. Clin. Chem. 66 (4), 549-555. doi:10.1093/clinchem/ hvaa029

Cooper, P. D. (1961). The Plaque Assay of Animal Viruses. Adv. Virus. Res. 8, 319-378. doi:10.1016/s0065-3527(08)60689-2

Cooper, P. D. (1955). A Method for Producing Plaques in agar Suspensions of Animal Cells. Virology 1 (4), 397-401. doi:10.1016/0042-6822(55)90033-7

Davis, F., and Higson, S. P. J. (2012). "Practical Applications and Protocols for Enzyme Biosensors," in Biosensors for Medical Application. Editor S. Higson (Woodhead Publishing Series in Biomaterails), 135-160. doi:10.1533/ 9780857097187.1.135

Diao, W., Tang, M., Ding, S., Li, X., Cheng, W., Mo, F., et al. (2018). Highly Sensitive Surface Plasmon Resonance Biosensor for the Detection of HIVRelated DNA Based on Dynamic and Structural DNA Nanodevices. Biosens. Bioelectron. 100, 228-234. doi:10.1016/j.bios.2017.08.042

Drobysh, M., Ramanaviciene, A., Viter, R., and Ramanavicius, A. (2021). Affinity Sensors for the Diagnosis of COVID-19. Micromachines 12 (4), 390. doi:10.3390/mi12040390

Duan, X., Huang, Y., Cui, Y., Wang, J., and Lieber, C. M. (2001). Indium Phosphide Nanowires as Building Blocks for Nanoscale Electronic and Optoelectronic Devices. Nature 409 (6816), 66-69. doi:10.1038/35051047

Garzón, V., Pinacho, D., Bustos, R.-H., Garzón, G., and Bustamante, S. (2019). Optical Biosensors for Therapeutic Drug Monitoring. Biosensors 9 (4), 132. doi:10.3390/bios9040132

Gong, Q., Yang, H., Dong, Y., and Zhang, W. (2015). A Sensitive Impedimetric DNA Biosensor for the Determination of the HIV Gene Based on Electrochemically Reduced Graphene Oxide. Anal. Methods 7 (6), 2554-2562. doi:10.1039/C5AY00111K

Gu, B., and Zhang, Q. (2018). Recent Advances on Functionalized Upconversion Nanoparticles for Detection of Small Molecules and Ions in Biosystems. Adv. Sci. 5 (3), 1700609. doi:10.1002/advs.201700609

Guliy, O. I., Zaitsev, B. D., Larionova, O. S., and Borodina, I. A. (2019). Virus Detection Methods and Biosensor Technologies. Biophysics 64 (6), 890-897. doi:10.1134/S0006350919060095

Kabir, M. A., Zilouchian, H., Younas, M. A., and Asghar, W. (2021). Dengue Detection: Advances in Diagnostic Tools from Conventional Technology to Point of Care. Biosensors 11 (7), 206. doi:10.3390/bios11070206

Kaushik, A., Kumar, R., Huey, E., Bhansali, S., Nair, N., and Nair, M. (2014). Silica Nanowires: Growth, Integration, and Sensing Applications. Microchim Acta 181 (15-16), 1759-1780. doi:10.1007/s00604-014-1255-0

Ktari, T., Baccar, H., Mejri, M. B., and Abdelghani, A. (2011). Calibration of Surface Plasmon Resonance Imager for Biochemical Detection. Int. J. Electrochemistry 2012, 1-5. doi:10.1155/2012/421692
La Spada, L., and Vegni, L. (2018). Electromagnetic Nanoparticles for Sensing and Medical Diagnostic Applications. Materials 11 (4), 603. doi:10.3390/ma11040603

Lee, D., Chander, Y., Goyal, S. M., and Cui, T. (2011). Carbon Nanotube Electric Immunoassay for the Detection of Swine Influenza Virus H1N1. Biosens. Bioelectron. 26 (8), 3482-3487. doi:10.1016/j.bios.2011.01.029

Lee, Y.-F., Lien, K.-Y., Lei, H.-Y., and Lee, G.-B. (2009). An Integrated Microfluidic System for Rapid Diagnosis of Dengue Virus Infection. Biosens. Bioelectron. 25 (4), 745-752. doi:10.1016/j.bios.2009.08.020

Leung, A., Shankar, P. M., and Mutharasan, R. (2007). A Review of Fiber-Optic Biosensors. Sensors Actuators B: Chem. 125 (2), 688-703. doi:10.1016/ J.SNB.2007.03.010

Li, J., Wu, D., Yu, Y., Li, T., Li, K., Xiao, M.-M., et al. (2021). Rapid and Unamplified Identification of COVID-19 with Morpholino-Modified Graphene Field-Effect Transistor Nanosensor. Biosens. Bioelectron. 183, 113206-206. doi:10.1016/ j.bios.2021.113206

Lisboa Bastos, M., Tavaziva, G., Abidi, S. K., Campbell, J. R., Haraoui, L.-P., Johnston, J. C., et al. (2020). Diagnostic Accuracy of Serological Tests for Covid-19: Systematic Review and Meta-Analysis. Bmj 370, m2516. doi:10.1136/bmj.m2516

Lu, C.-H., Zhang, Y., Tang, S.-F., Fang, Z.-B., Yang, H.-H., Chen, X., et al. (2012). Sensing HIV Related Protein Using Epitope Imprinted Hydrophilic Polymer Coated Quartz crystal Microbalance. Biosens. Bioelectron. 31 (1), 439-444. doi:10.1016/j.bios.2011.11.008

Monteiro, T., and Almeida, M. G. (2019). Electrochemical Enzyme Biosensors Revisited: Old Solutions for New Problems. Crit. Rev. Anal. Chem. 49 (1), 44-66. doi:10.1080/10408347.2018.1461552

Ngo, H. T., Wang, H.-N., Fales, A. M., Nicholson, B. P., Woods, C. W., and VoDinh, T. (2014). DNA Bioassay-On-Chip Using SERS Detection for Dengue Diagnosis. Analyst 139 (22), 5655-5659. doi:10.1039/c4an01077a

Nidzworski, D., Siuzdak, K., Niedziałkowski, P., Bogdanowicz, R., Sobaszek, M., Ryl, J., et al. (2017). A Rapid-Response Ultrasensitive Biosensor for Influenza Virus Detection Using Antibody Modified boron-doped diamond. Sci. Rep. 7 (1). doi:10.1038/s41598-017-15806-7

Pang, Y., Rong, Z., Wang, J., Xiao, R., and Wang, S. (2015). A Fluorescent Aptasensor for H5N1 Influenza Virus Detection Based-On the Core-Shell Nanoparticles Metal-Enhanced Fluorescence (MEF). Biosens. Bioelectron. 66, 527-532. doi:10.1016/j.bios.2014.10.052

Peduru Hewa, T. M., Tannock, G. A., Mainwaring, D. E., Harrison, S., and Fecondo, J. V. (2009). The Detection of Influenza A and B Viruses in Clinical Specimens Using a Quartz crystal Microbalance. J. Virol. Methods 162 (1-2), 14-21. doi:10.1016/j.jviromet.2009.07.001

Prabowo, B., Purwidyantri, A., and Liu, K.-C. (2018). Surface Plasmon Resonance Optical Sensor: A Review on Light Source Technology. Biosensors 8 (3), 80. doi:10.3390/bios 8030080

Qi, C., Tian, X.-S., Chen, S., Yan, J.-H., Cao, Z., Tian, K.-G., et al. (2010). Detection of Avian Influenza Virus Subtype H5 Using a Biosensor Based on Imaging Ellipsometry. Biosens. Bioelectron. 25 (6), 1530-1534. doi:10.1016/j.bios.2009.10.030

Qiu, G., Gai, Z., Tao, Y., Schmitt, J., Kullak-Ublick, G. A., and Wang, J. (2020). Dual-Functional Plasmonic Photothermal Biosensors for Highly Accurate Severe Acute Respiratory Syndrome Coronavirus 2 Detection. ACS nano 14 (5), 5268-5277. doi:10.1021/acsnano.0c02439

Rettcher, S., Jungk, F., Kühn, C., Krause, H.-J., Nölke, G., Commandeur, U., et al. (2015). Simple and Portable Magnetic Immunoassay for Rapid Detection and Sensitive Quantification of Plant Viruses. Appl. Environ. Microbiol. 81 (9), 3039-3048. doi:10.1128/AEM.03667-14

Scholz, F. (2015). Voltammetric Techniques of Analysis: the Essentials. ChemTexts 1, 17. doi:10.1007/s40828-015-0016-y

Seo, G., Lee, G., Kim, M. J., Baek, S.-H., Choi, M., Ku, K. B., et al. (2020). Rapid Detection of COVID-19 Causative Virus (SARS-CoV-2) in Human Nasopharyngeal Swab Specimens Using Field-Effect Transistor-Based Biosensor. ACS nano 14 (4), 5135-5142. doi:10.1021/acsnano.0c02823

Shamsipur, M., Nasirian, V., Mansouri, K., Barati, A., Veisi-Raygani, A., and Kashanian, S. (2017). A Highly Sensitive Quantum Dots-DNA Nanobiosensor Based on Fluorescence Resonance Energy Transfer for Rapid Detection of Nanomolar Amounts of Human Papillomavirus 18. J. Pharm. Biomed. Anal. 136, 140-147. doi:10.1016/j.jpba.2017.01.002

Shoaie, N., Daneshpour, M., Azimzadeh, M., Mahshid, S., Khoshfetrat, S. M., Jahanpeyma, F., et al. (2019). Electrochemical Sensors and Biosensors Based on 
the Use of Polyaniline and its Nanocomposites: a Review on Recent Advances. Microchim Acta 186 (7), 465. doi:10.1007/s00604-019-3588-1

Singh, P., Pandey, S. K., Singh, J., Srivastava, S., Sachan, S., and Singh, S. K. (2016). Biomedical Perspective of Electrochemical Nanobiosensor. Nano-micro Lett. 8 (3), 193-203. doi:10.1007/s40820-015-0077-x

Souza, E., Nascimento, G., Santana, N., Ferreira, D., Lima, M., Natividade, E., et al. (2011). Label-free Electrochemical Detection of the Specific Oligonucleotide Sequence of Dengue Virus Type 1 on Pencil Graphite Electrodes. Sensors 11 (6), 5616-5629. doi:10.3390/s110605616

Wang, J., Cai, X., Rivas, G., Shiraishi, H., Farias, P. A. M., and Dontha, N. (1996). DNA Electrochemical Biosensor for the Detection of Short DNA Sequences Related to the Human Immunodeficiency Virus. Anal. Chem. 68 (15), 2629-2634. doi:10.1021/ac9602433

Wang, S., Li, L., Jin, H., Yang, T., Bao, W., Huang, S., et al. (2013). Electrochemical Detection of Hepatitis B and Papilloma Virus DNAs Using SWCNT Array Coated with Gold Nanoparticles. Biosens. Bioelectron. 41, 205-210. doi:10.1016/ j.bios.2012.08.021

Wang, Y., Chen, M., Zhang, L., Ding, Y., Luo, Y., Xu, Q., et al. (2009). Rapid Detection of Human Papilloma Virus Using a Novel Leaky Surface Acoustic Wave Peptide Nucleic Acid Biosensor. Biosens. Bioelectron. 24 (12), 3455-3460. doi:10.1016/j.bios.2009.04.034

Wu, G., Meyyappan, M., and Lai, K. (2018). Simulation of Graphene Field-Effect Transistor Biosensors for Bacterial Detection. Sensors 18 (6), 1715. doi:10.3390/ s18061715

Xu, L., Yu, H., Akhras, M. S., Han, S.-J., Osterfeld, S., White, R. L., et al. (2008). Giant Magnetoresistive Biochip for DNA Detection and HPV Genotyping. Biosens. Bioelectron. 24 (1), 99-103. doi:10.1016/j.bios.2008.03.030

Xu, M., Wang, R., and Li, Y. (2016). An Electrochemical Biosensor for Rapid Detection of E. coli O157:H7 with Highly Efficient Bi-functional Glucose
Oxidase-Polydopamine Nanocomposites and Prussian Blue Modified Screen-Printed Interdigitated Electrodes. Analyst 141 (18), 5441-5449. doi:10.1039/c6an00873a

Zaytseva, N. V., Montagna, R. A., and Baeumner, A. J. (2005). Microfluidic Biosensor for the Serotype-specific Detection of Dengue Virus RNA. Anal. Chem. 77 (23), 7520-7527. doi:10.1021/ac0509206

Zhang, H., Liu, L., Li, C.-W., Fu, H., Chen, Y., and Yang, M. (2011). Multienzyme-nanoparticles Amplification for Sensitive Virus Genotyping in Microfluidic Microbeads Array Using Au Nanoparticle Probes and Quantum Dots as Labels. Biosens. Bioelectron. 29 (1), 89-96. doi:10.1016/ j.bios.2011.07.074

Conflict of Interest: The authors declare that the research was conducted in the absence of any commercial or financial relationships that could be construed as a potential conflict of interest.

Publisher's Note: All claims expressed in this article are solely those of the authors and do not necessarily represent those of their affiliated organizations, or those of the publisher, the editors and the reviewers. Any product that may be evaluated in this article, or claim that may be made by its manufacturer, is not guaranteed or endorsed by the publisher.

Copyright (C) 2022 Shand, Dutta, Rajakumar, James Paulraj, Mandal, KT and Ghorai. This is an open-access article distributed under the terms of the Creative Commons Attribution License (CC BY). The use, distribution or reproduction in other forums is permitted, provided the original author(s) and the copyright owner(s) are credited and that the original publication in this journal is cited, in accordance with accepted academic practice. No use, distribution or reproduction is permitted which does not comply with these terms. 